\title{
Perception and Extrapolation of Velocity and Acceleration
}

\author{
David A. Rosenbaum \\ Stanford University
}

\begin{abstract}
A moving target disappeared behind a screen and subjects predicted when the target passed behind a marker on the screen. When the target moved with constant velocity, predictions were extremely accurate, regardless of the spatial and temporal exposure and concealment of the target and regardless of its rate of velocity. When the target accelerated, accuracy of prediction decreased with increasing acceleration and with increasing target concealment. Analyses of the results suggest that the perception of velocity and acceleration is direct and accurate and that extrapolation of velocity and acceleration incorporates concrete and abstract characteristics of the motion that was seen. It is proposed that the motion perception system is tuned to accelerated rather than to constant velocity movement.
\end{abstract}

It is often necessary to predict where a moving object will be at some time, when it will be at some place, or a combination of the two. In all such cases at least three things must happen. First, the observer must perceptually determine the rate and path of the object's motion. Second, the observer must extrapolate the motion that was seen. Third, initiation of a response associated with the motion must take into account the spatial and temporal requirements of the response and the spatial and temporal remainder of the motion. One activity which depends on these three processes is the batting of a baseball. The batter determines the speed and trajectory of the oncoming ball in a relatively short time. Then he determines the eventual location of the ball and the time until it passes his body. Finally, he initiates the swing of his bat at precisely the moment when it will be possible for the bat to meet the ball. All of these events occur in a highly integrated

The research was conducted while the author was supported by a National Science Foundation Graduate Fellowship. Thanks are extended to Michael Radford for his research assistance, Martin LePoint of the Stanford Physics Department for supplying the linear air track, Gordon Bower, Roger Shepard, Judith Kroll, and the editors of this journal for comments on earlier versions of this paper, and to Arnold Glass for his support and counsel.

Requests for reprints should be sent to David A. Rosenbaum, Department of Psychology, Stanford University, Stanford, California 94305. sequence. They illustrate the complexity of motion prediction and the sophistication required for its successful use.

In most cases of motion prediction the object being observed accelerates or decelerates and only rarely moves with constant velocity. Yet almost all research on the perception of motion has used constant velocity displacement. Wundt (1874) and Geiger (1903) studied acceleration perception, but for methodological reasons their results were inconclusive. Gottsdanker (1955) studied manual tracking of an accelerated target in a random two-dimensional path. After the target disappeared behind a screen, subjects continued to track the target at its average exposed velocity. Unfortunately, Gottsdanker did not determine whether this result was due to a failure in perception or extrapolation, limitations in motor control, or the complexity of the path traversed by the target. Thus, the basic question remains: How accurately can human observers determine rates of visually perceived acceleration? To distinguish acceleration perception from motion perception generally, the same question may be asked about constant velocity motion. If acceleration and constant velocity are perceived with equally high accuracy, this would suggest that in perception as well as in physics constant velocity may be regarded as a special case of acceleration-the case in which acceleration is equal to zero. 
To study the accuracy of velocity and acceleration perception, a simplified version of Gottsdanker's (1955) experiment was conducted. A target moving horizontally disappeared behind a screen and subjects pressed a button when they thought the target passed directly behind a marker on the screen. The distance and time covered by the target in its exposed motion were varied, as were the distance and time covered by the target in its hidden motion to the point behind the marker. The target moved at three rates of constant velocity in Experiment 1 and at three rates of uniform acceleration in Experiment 2. By recording subjects' time estimations, it was possible to determine how accurately they could determine the target's rate of velocity and acceleration and how accurately they could extrapolate these two kinds of motion.

By varying the target's exposed distance and time, it was possible to answer a second question about the perception of motion: Is the perception of velocity and, in particular, acceleration direct or indirect? If the perception of motion is indirect, it is presumed that the observer infers motion by noting positional changes of external objects and the time in which these changes occur. If the perception of motion is direct, it is presumed that information about motion per se is coded in the visual system. A number of psychophysical (Pantle \& Sekuler, 1968; Sekuler \& Ganz, 1963) and physiological (Barlow, Hill, \& Levick, 1964; Hubel \& Wiesel, 1968; Lettvin, Maturana, McCulloch, \& Pitts, 1959) experiments suggest that such coding occurs. It is not known, however, whether the coding of velocity differs from the coding of acceleration.

To resolve this point we may draw on the definitions of velocity and acceleration. Velocity is defined as the rate of change of position in time. Let us assume, then, that if an observer did not perceive velocity directly, he would have to process positional and temporal information separately to infer a particular velocity rate. It is reasonable that if velocity were inferred in this way, the accuracy of an observer's inference would have a greater chance of being influenced by particular values of distance and time than if velocity were perceived directly. In Experiment 1 , therefore, if the accuracy of subjects' perceptions are markedly influenced by differences in the exposed distance and time of the target's motion, this would suggest that velocity is not perceived directly. But if the accuracy of subjects' perceptions are relatively uninfluenced by differences in the exposure of the target, this would suggest that the perception of velocity is direct.

A similar argument applies to the perception of acceleration. Acceleration is defined as the rate of change of the rate of change of position in time. If an observer did not perceive acceleration directly, he would have to process positional and temporal information separately on at least two occasions to infer a particular acceleration rate. The rate actually inferred would depend on the degree of difference between the positional and temporal information processed on the two occasions. Following the same assumptions as were made in regard to velocity perception, if the accuracy of subjects' perceptions in Experiment 2 is markedly influenced by differences in the exposed distance and time of the target's motion, this would suggest that acceleration is not perceived directly. But if the accuracy of subjects' perceptions is relatively uninfluenced by differences in the exposure of the target, this would suggest that the perception of acceleration is direct. Of course, it is recognized that in conditions of very limited exposure the accuracy of subjects' perceptions of velocity and acceleration could decline sharply, whether the perceptions were direct or not. But if this decline did not occur, it would suggest even more strongly that the perception of velocity and acceleration is direct.

A third question addressed in the present research was: How accurately and by what general methods do human observers extrapolate the motion they have seen? To extrapolate constant velocity, subjects could adopt one of two general strategies. They could determine the ratio of exposed distance to hidden distance, time the movement of the target through the exposed distance, and then "compute" the time required to 
traverse the hidden distance according to the ratio they found before. If this strategy were used, subjects' performance would probably deteriorate when the ratio of exposed distance to hidden distance was very small or not a common fraction. In addition, eye movements during the hidden motion would tend to be relatively independent of the actual motion of the target behind the screen. A second strategy that subjects could adopt is to extend directly the motion they saw, perhaps by relying on a form of imagery. If this strategy were used, subjects' performance would probably be relatively unaffected by the ratio of exposed distance to hidden distance, and eye movements during extrapolation would tend to mirror the hidden motion of the target.

While either of these strategies could be used for extrapolating constant velocity, neither would suffice for extrapolating acceleration. If subjects extrapolated acceleration by computing hidden time according to the ratio of exposed distance to hidden distance, the necessary computations would be extremely complex and extrapolation would probably fail. If subjects directly extended the motion they saw without tracking at a different speed from the visible speed of the target, they would severely overestimate the hidden time of the target's motion. To extrapolate acceleration properly, subjects would have to speed up their implicit tracking of the hidden target at a rate specified by its visible acceleration. That is, they would have to determine the target's rate of change of velocity and apply this rate of change first to the terminal exposed velocity of the target and then to subsequent velocities attributed to the target during its hidden motion.

\section{EXPERIMENT 1}

\section{Method}

Design. Three constant values of distance and time were selected for the exposed and hidden portions of the target's movement. These values are presented in Table 1. The values of exposed distance and exposed time were expected to be sensitive determinants of subjects' discrimination; the shortest exposed distance was also the diameter of the target. The values of hidden distance and hidden time were expected to be
TABLE 1

Constant Values for Exposed Distance and Time and Hidden Distance AND TIME

\begin{tabular}{cccc}
$\begin{array}{c}\text { Exposed } \\
\text { distance } \\
(\mathrm{cm})\end{array}$ & $\begin{array}{c}\text { Exposed } \\
\text { time } \\
(\mathrm{sec})\end{array}$ & $\begin{array}{c}\text { Hidden } \\
\text { distance } \\
(\mathrm{cm})\end{array}$ & $\begin{array}{c}\text { Hidden } \\
\text { time } \\
(\mathrm{sec})\end{array}$ \\
\hline 3.5 & .25 & 20.0 & .50 \\
7.0 & .50 & 40.0 & .75 \\
10.5 & .75 & 60.0 & 1.00 \\
\hline
\end{tabular}

large enough to require more than an instantaneous response to the disappearance of the target but not so large that extrapolation would become extremely inaccurate.

The three rates of velocity were $18.76 \mathrm{~cm} / \mathrm{sec}$, $42.90 \mathrm{~cm} / \mathrm{sec}$, and $72.72 \mathrm{~cm} / \mathrm{sec}$. These rates were selected primarily on the basis of the capabilities of the experimental apparatus.

Exposure and concealment conditions were grouped as follows. Each of the three exposed distances was paired with each of the three hidden distances, allowing exposed time and hidden time to vary with velocity. Each of the three exposed distances was also paired with each of the three hidden times, allowing exposed time and hidden distance to vary with velocity. Similarly, each of the three exposed times was paired with each of the three hidden distances, allowing exposed distance and hidden time to vary with velocity. And each of the three exposed times was paired with each of the three hidden times, allowing exposed distance and hidden distance to vary with velocity. There were 36 presentations at each velocity and a total of 108 presentations for each subject.

Apparatus. The main apparatus was a linear air track obtained from the Stanford Physics Department. A linear air track is a straight metal runway, shaped like an inverted $V$. Air is blown with a shop-type blower (Sears Model No. 758169002 ) through holes in the runway. When a metal sled is placed on top of the runway it rides on the air and so is acted upon by a negligible amount of friction. The air track that was used in the present experiment was $1.38 \mathrm{~m}$ long and had a slingshot device on one end which allowed for accurate launching of the target. Masking tape was placed on the opposite end of the track to reduce the sound of the target reaching the end of the runway. Because the blower was noisy, it further prevented subjects from hearing the target at the end of the track. However, subjects (and the experimenter) quickly got used to the sound of the blower.

The track was hidden behind a wooden framework made up of a large section $(29.2 \times 51.4 \mathrm{~cm})$ that concealed the slingshot device and the experimenter's hand as he launched the target, and a longer section $(20.9 \times 192.4 \mathrm{~cm})$ that concealed the major portion of the air track but left the target 
in view. A grooved board $(3.4 \times 3.4 \times 192.4 \mathrm{~cm})$ attached to the base of the long section of the framework allowed a screen $(20.9 \times 192.4 \mathrm{~cm})$ to be moved across the front of the air track in such a way that it concealed the target for any specified distance. A white cardboard marker $(3.5 \times 7.3$ $\mathrm{cm}$ ) was placed over the top edge of the screen and was movable across it. The width of the marker was identical to the diameter of the target.

The target was a table-tennis ball mounted on cardboard which was placed on a metal sled desigred to ride on the air track. The target was launched at different velocities by pulling the sled against the elastic band over different predetermined distances. Immediately before appearing from behind the large section of the framework, the target (or its cardboard mounting) interrupted a light beam in a Knight photoelectric relay. This interruption triggered a clock (Lafayette Model 54519) which was stopped when the subject pressed a button on a simple hand-held response box. Because of the construction of the apparatus, the target always moved from left to right.

The subject sat $168 \mathrm{~cm}$ from the apparatus, directly opposite the exit point of the target and with his eyes level with the air track.

Subjects. Four males and two females volunteered as subjects. Five subjects were psychology graduate students and one subject was a member of the Stanford Psychology Department. None of the subjects reported having any perceptual or motor disabilities.

Procedure. Subjects were informed that they were participating in a study of motion perception. They were instructed to press the response button at exactly the moment when they thought the object passed directly behind the marker.

To familiarize subjects with the procedure and to obtain their baselines, the marker was placed on the vertical edge of the screen closest to the launching site. Ten trials were conducted in this fashion at each velocity, with an exposed distance of $36.36 \mathrm{~cm}$.

At the start of each experimental trial, the experimenter set the screen and marker at distances indicated on a card containing only this information and the presentation velocity. After retracting the sled to the exact launch position, the experimenter gave the warning, "Ready," and released the sled.

The order of trials was randomized for each subject by shufling the cards before each session. No feedback was provided to subjects about the accuracy of their time estimations either during the familiarization period or during the actual experiment.

Rests were taken whenever the subject or experimenter requested it. On the average, a total of three rests was taken. The experiment lasted about $75 \mathrm{~min}$.

\section{Results}

The results suggest that subjects perceived velocity directly and accurately and that extrapolation of velocity was performed accurately through a process of direct extension. These conclusions were drawn from the following analysis. The recorded time for each subject on each trial was converted to a subjective velocity by dividing the recorded time into the total distance (i.e., sum of exposed distance and hidden distance) covered by the target on that trial. Then the subjective velocities for each trial were averaged across subjects. A regression analysis was performed, using average subjective velocity as the dependent measure and exposed distance, exposed time, hidden distance, hidden time, and objective velocity as the independent measures.

According to the hypothesis presented earlier, if velocity is not perceived directly, exposed distance and exposed time should account for much of the variance in subjective velocity, but if velocity is perceived directly, exposed distance and exposed time should account for very little of this variance. The latter prediction held up. After partialing out hidden distance, hidden time, and objective velocity, exposed distance accounted for only $.81 \%$ of the variance in subjective velocity and exposed time accounted for only $1.96 \%$ of the variance in subjective velocity.

This result could attest to a complete failure of extrapolation rather than to the directness and accuracy of perception. If so, hidden distance and hidden time would be expected to account for most of the variance in subjective velocity. It was found, however, that after partialing out exposed distance, exposed time, and objective velocity, hidden distance accounted for only $1.21 \%$ of the variance in subjective velocity and hidden time accounted for only $.16 \%$ of this variance. When objective velocity alone was correlated with subjective velocity it was found to account for $90.25 \%$ of the variance in subjective velocity. This high correlation resulted from the extreme accuracy of subjects' time estimations. Generally, estimated times lagged behind actual 
times by an amount that was equivalent to the response latencies (about 140 to 250 msec) recorded in the baseline condition of the experiment.

These results indicate that the perception of velocity was direct and accurate and that extrapolation was performed efficiently. Moreover, because hidden distance accounted for so little variance in subjective velocity, it is unlikely that subjects extrapolated velocity by computing hidden time according to the ratio of exposed distance to hidden distance. Rather, it seems that extrapolation was achieved by directly extending the motion that was seen. Evidence for direct extension derives from informal observations of subjects' eye movements during the hidden motion of the target. Most often the eyes moved continuously as if the target were still in view. Sometimes the eyes moved in regular intervals over the screen in the direction of the marker. Only rarely did subjects' eyes move directly to the marker and fixate it until responding. Unfortunately, these differences in eye movements were not consistent across subjects or presentation conditions, so that the effects of different eye-movement patterns on the accuracy of time estimation cannot be determined.

\section{Experiment 2}

The second experiment investigated the perception and extrapolation of uniformly accelerated motion. Because it was not known before doing the experiment how well people could recognize acceleration, it was decided that half the subjects would be told that the target would speed up in all presentations. It was assumed that by telling subjects to expect acceleration, some of the processing needed to infer acceleration would be made unnecessary for them. Thus, if the informed subjects performed better than the uninformed subjects, this would support the idea that acceleration is normally inferred. On the other hand, if informed and uninformed subjects performed equally, this would support the idea that acceleration is perceived directly.

\section{Method}

Design. The design of the experiment was identical to that of Experiment 1. Some of the hidden distances required by the preassigned hidden times were greater than the length of the screen. These trials were omitted, leaving a total of 91 trials.

Subjects were informed or uninformed depending on their background in physics. Subjects who had never studied physics were assigned to the uninformed group and those who had studied physics were assigned to the informed group. The reason for this assignment strategy was that sophisticated subjects would be able to reason that acceleration occurred on the basis of the presentation procedure.

Apparatus. To vary the acceleration of the target, different weights were attached to the sled and another weight, attached to the sled with 3 -in. Scotch-brand recording tape, was suspended over a frictionless metal arc at the end of the track. The three acceleration rates obtained in this way were empirically measured as $23.30 \mathrm{~cm} /$ $\mathrm{sec}^{2}, 39.37 \mathrm{~cm} / \mathrm{sec}^{2}$, and $43.81 \mathrm{~cm} / \mathrm{sec}^{2}$. The weight at the end of the track was concealed in all aspects of its movement. Because the target started from rest while in view, it was necessary to fit a second cardboard mounting on the sled in such a way that the photoelectric beam would pass between the cardboards before the movement and would be interrupted by the second carboand immediately after the movement started.

Subjects. Six male and two female Stanford students volunteered as subjects. None of the subjects reported any perceptual or motor problems.

Procedure. Before each presentation, the experimenter added or removed weights from the sled behind the large portion of the framework. Next, the sled was positioned in such a way that the target stood at rest immediately to the subject's right of the exit point. Movement started when the experimenter let go of the sled. At the start of the experiment, informed subjects were told that the target would always speed up and uninformed subjects were told nothing about the target's motion.

\section{Results}

The results suggest that subjects perceived acceleration directly and accurately and that their extrapolations incorporated acceleration information. Because a number of analyses were performed to reach this conclusion, a brief overview of the analyses will be helpful for the reader. It will be demonstrated here (a) that subjects perceived acceleration and were not simply responding to the average exposed velocity or to the terminal exposed velocity of the target, (b) that exposed distance and ex- 
TABLE 2

Variance in Subjective Acceleration ACCOUNTED FOR BY TIMES BASED ON OBJECTIVE ACCELERATTON AND Average Exposed Velocity

\begin{tabular}{lcc}
\hline Acceleration rate & $\begin{array}{c}\text { Objective } \\
\text { acceleration }\end{array}$ & $\begin{array}{c}\text { Average exposed } \\
\text { velocity }\end{array}$ \\
\hline $\begin{array}{l}\text { Slow } \\
\text { Uninformed subject }\end{array}$ & .81 & .42 \\
$\quad$ Informed subject & .92 & .45 \\
Medium & & \\
$\quad$ Uninformed subject & .49 & .25 \\
$\quad$ Informed subject & .86 & .54 \\
Fast & & \\
$\quad$ Uninformed subject & .07 & .89 \\
$\quad$ Informed subject & .03 & .78 \\
\hline
\end{tabular}

posed time accounted for very little variance in subjective acceleration and that this result was not due to the possibility that extrapolation was so inaccurate that it accounted for a plethora of variance in subjective acceleration, (c) that the perception of acceleration was nearly veridical, and (d) that the accuracy of extrapolation depended on an interaction of objective acceleration and concealment of the target.

As in Experiment 1, a regression analysis was performed to find the main determinants of subjects' time estimations. For each trial and for every subject a subjective acceleration score $(a)$ was obtained by dividing the square of the recorded time $(t)$ into twice the total distance $(s)$ covered by the target $\left(a=2 s / t^{2}\right)$. Subjective accelerations were then averaged across subjects within each group for every trial. A multiple regression was performed for each group using average subjective acceleration as the dependent variable and exposed distance, exposed time, hidden distance, hidden time, and objective acceleration as the independent variables.

First, we consider only the correlations between objective acceleration and subjective acceleration in the two groups. The correlation was .55 in the informed group and .54 in the uninformed group. Since the correlations were positive, subjects either recognized acceleration or merely speeded up their extrapolations. The latter explanation can be dismissed, for in Experiment 1, where subjects did not see acceleration, extrapolations did not speed up.

To make sure that subjects were actually recognizing acceleration and were not simply responding to the average exposed velocity of the target or to its terminal exposed velocity, the following analysis was performed. For each trial, two theoretical times were computed. One was the time required for the target to reach the marker site at the objective rate of acceleration. The other was the time required for the target to cover this same distance at its average exposed velocity. These values were correlated in a multiple regression with the average recorded time for each group on every trial. Since terminal exposed velocity and average exposed velocity are linearly related, only the theoretical times based on one of these measures were required in the analysis. The results appear in Table 2 . It is seen here that times based on objective acceleration accounted for more of the variance in obtained times than did times based on average exposed velocity. This was true for both groups of subjects in two of the three acceleration conditions. Only at the fastest rate of acceleration were obtained times better predicted by average exposed velocity than by objective acceleration. It can be concluded, therefore, that both groups recognized acceleration and extrapolated in an accelerated way at the slow and medium accelerations rates.

Next, we can determine whether acceleration was inferred or perceived directly in the slow and medium and also in the fast acceleration conditions. Because the correlations between subjective acceleration and objective acceleration were about equal for the informed and uninformed subjects, we would suppose on the basis of our previous assumptions that acceleration is perceived directly. Support for this inference derives from the multiple regression described earlier. As is seen in Table 3, the correlations between subjective acceleration and exposed distance and time increased as acceleration grew faster. Even at the fastest acceleration, however, exposure conditions accounted for only $.47^{2}$ or $22 \%$ of the vari- 
TABLE 3

Correlations Between Subjective Acceleration and Objective Acceleration (OA), Exposed Distance (ED), Exposed Time (ET), Hidnen Distance (HD), AND HidDEN TIME (HT)

\begin{tabular}{lcccccccccc}
\hline & \multicolumn{4}{c}{ Uninformed subject } & \multicolumn{4}{c}{ Informed subject } \\
Rate of OA & OA & ED & ET & HD & HT & OA & ED & ET & HD & HT \\
\hline Slow & .00 & .03 & .03 & -.71 & -.71 & .00 & .13 & .13 & -.60 & -.60 \\
Medium & .00 & .25 & .25 & -.85 & -.85 & .00 & .29 & .29 & -.74 & -.74 \\
Fast & .08 & .47 & .47 & -.76 & -.76 & .16 & .56 & .56 & -.52 & -.52 \\
Overall & .54 & .14 & .01 & -.55 & -.70 & .55 & .21 & .07 & -.37 & -.58 \\
\hline
\end{tabular}

ance in subjective acceleration for uninformed subjects and $.56^{2}$ or $31 \%$ of the variance in subjective acceleration for informed subjects. In all conditions, except in the fastest acceleration rate for informed subjects, exposed distance and time correlated less with subjective acceleration than did hidden distance and time. Across all acceleration rates, where the effects of distance and time could be tested separately, the correlations between subjective acceleration and exposed distance and time were quite small and were much smaller than the correlations between subjective acceleration and hidden distance and time. Stepwise regression on the final row of Table 3 demonstrates that, in general, exposed distance and exposed time accounted for only a slight amount of variance in subjective acceleration. These results appear in Table 4. Here it is seen that hidden time accounted for most of the variance in subjective acceleration and that successively smaller amounts of variance were accounted for by objective acceleration, exposed distance, exposed time, and hidden distance. These results are consistent with the hypothesis that acceleration is perceived directly. However, they do not show conclusively that the hypothesis is correct. It is possible that extrapolation of acceleration was simply so poor that it accounted for most of the variance in subjective acceleration.

Inspection of Figure 1 reveals that this is not the case. The vertical axis of the figure represents the accuracy of subjective acceleration, defined as the ratio of subjective acceleration to objective acceleration. The horizontal axis represents the three values of hidden distance held constant throughout the experiment. At the shortest hidden distance, the accuracy of subjective acceleration was extremely high. Since performance at this distance required a minimal amount of extrapolation, the accuracy of subjective acceleration here provides a relatively pure measure of perceptual accuracy. It is evident from the figure, therefore, that perception of acceleration was extremely accurate at all rates of objective acceleration. Given that exposed distance and exposed time accounted for so little variance in subjective acceleration, it then follows that the perception of acceleration was direct.

Apparently, the accuracy of subjects' time estimations was almost completely determined by extrapolation. Judging from the curves in Figure 1, it appears that accuracy declined as longer extrapolations were required and as acceleration increased. An analysis of variance tested this judgment. The analysis tested the effects of objective acceleration, exposed distance, hidden dis-

TABLE 4

Relative Contribution of Each Factor to the Variance in Subjective Acceleration

\begin{tabular}{lcccccc}
\hline & \multicolumn{2}{c}{ Uninformed subject } & \multicolumn{3}{c}{ Informed subject } \\
$\begin{array}{c}\text { Factor } \\
\text { entered }\end{array}$ & $\begin{array}{c}\text { Mul- } \\
\text { tiple }\end{array}$ & $r^{2}$ & $\begin{array}{c}\text { Clange } \\
\text { in } r^{2}\end{array}$ & $\begin{array}{c}\text { Mul- } \\
\text { tiple } \\
r\end{array}$ & $r^{2}$ & $\begin{array}{c}\text { Change } \\
\text { in } r^{2}\end{array}$ \\
\hline $\begin{array}{l}\text { Hidden time } \\
\text { Objective ac- } \\
\text { celeration }\end{array}$ & .70 & .49 & .49 & .58 & .33 & .33 \\
$\begin{array}{c}\text { Exposed } \\
\text { distance }\end{array}$ & .81 & .65 & .01 & .73 & .53 & .01 \\
$\begin{array}{c}\text { Exposed } \\
\text { time }\end{array}$ & .82 & .67 & .02 & .74 & .55 & .02 \\
$\begin{array}{c}\text { Hidden } \\
\text { distance }\end{array}$ & .82 & .67 & .00 & .75 & .56 & .01 \\
\hline
\end{tabular}

Note. Multiple $\gamma=$ coefficient of correlation between all factors entered and subjective acceleration, $r^{2}=$ variance in subjective acceleration accounted for by all factors entered. 


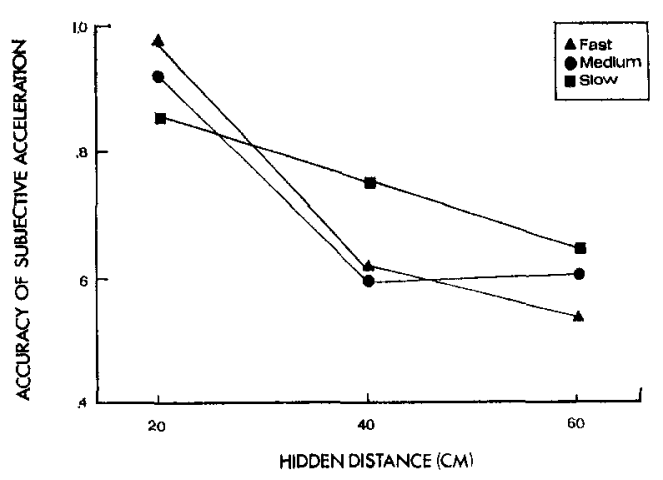

FIGURE 1. Accuracy of subjective acceleration for fast, medium, and slow rates of objective acceleration at three hidden distances.

tance, state of informedness, and subjects on the accuracy of subjective acceleration. Exposed time and hidden time were omitted from the analysis because they are perfectly confounded with exposed distance and hidden distance, respectively, at each acceleration rate. A significant interaction emerged between hidden distance and objective acceleration, $F(2,24)=3.82, p<$ .05 ; for longer hidden distances and at higher rates of acceleration the accuracy of subjective acceleration declined sharply. Hidden distance had a main effect on the accuracy of subjective acceleration, $F(2,12)$ $=5.34, p<.05$; as hidden distance increased, accuracy declined. There was also a main effect of objective acceleration, $F(2$, $12)=20.78, p<.01$; with higher objective accelerations, subjective acceleration became less accurate. No other effects were significant. An interpretation of these results is clear. As acceleration increases, the rate of change of tracking speed must increase, and as longer extrapolation is required, the change in the absolute speed of tracking must also increase. At the fastest acceleration rate, therefore, the demands of extrapolation were greater than at slower rates of acceleration. Accordingly, the fact that subjects' time estimations in the fastest acceleration condition were better predicted by average exposed velocity than by objective acceleration (see Table 2) can be attributed to difficulties subjects had in ex- trapolating acceleration and not to difficulties which they had in seeing acceleration in the first place.

Subjects' eye movements in the extrapolation of acceleration were similar to subjects' eye movements in the extrapolation of constant velocity. Generally, subjects' eyes moved continuously as if the target were still in view. Eye movements and head rotations showed an unmistakable pattern of acceleration. One pattern of eye movements was particularly interesting. On some trials subjects' eyes moved at regular intervals and covered successively longer distances during each saccade. As before, visual tracking patterns were not consistent across subjects or trials, so that their effects on the accuracy of performance cannot be assessed.

\section{DISCUSSION}

These experiments suggest that human subjects perceive velocity and acceleration directly and accurately. The experiments also suggest that extrapolation of velocity is performed accurately through a direct extension of the movement that was seen. The extrapolation of acceleration was found to deteriorate as acceleration increased and as concealment grew longer. Subjects' eye movements during the extrapolation of acceleration appeared to mirror the general kinematic properties of the target.

These results have two main theoretical implications. The first is that, because velocity and acceleration were both perceived directly and accurately, constant velocity may be regarded as a special case of acceleration in perception as well as in physics. We might speculate further that the motion perception system is primarily turned to acceleration rather than to constant velocity. If, as a number of theorists have claimed. the perceptual system is primarily responsive to changes in stimulation, then it is noteworthy that acceleration involves more changes than does constant velocity. Constant velocity involves changes of position in time while acceleration involves changes in these changes of position in time. From the standpoint of responsive- 
ness to change, therefore, acceleration is more evocative than constant velocity and may, for that reason, be more readily registered in the nervous system. This speculation is made plausible by the sheer commonness of acceleration in the physical world, in contrast to the relative rarity of sustained constant velocity motion.

A second theoretical implication of the present results concerns the abstract, relational information that is conveyed by acceleration. Although acceleration necessarily occurs when velocity changes, a particular rate of acceleration may remain invariant over many changes in velocity. Acceleration thus defines an abstract relation among an infinite number of velocities. Because extrapolation of acceleration demands the application of this relation to different tracking speeds, the fact that subjects in Experiment 2 could extrapolate accurately demonstrates that they obtained such an abstract relation while viewing the motion of the target.

\section{REFERENCES}

Barlow, H. B., Hill, R. M., \& Levick, W. R. Retinal ganglion cells responding selectively to direction and speed of image motion in the rabbit. Journal of Physiology, 1964, 173, 377407.

Geiger, M. Neue complicationsversuche. Psychologische Studien, 1903, 18, 347-436.

Gottsdanker, R. M. A further study of predictionmotion. American Journal of Psychology, $1955,68,432-437$.

Hubel, D. H., \& Wicsel. T. N. Receptive fields and functional architecture of monkey striate cortex. Journal of Physiology, 1968, 195, 215243.

Lettvin, J. Y., Maturana, H. R., McCulloch, W. S., \& Pitts, W. H. What the frog's eye tells the frog's brain. Proceedings of the Institute of Radio Engineers, 1959, 47, 1940-1951.

Pantle, A. J., \& Sekuler, R. W. Velocity-sensitive elements in human vision: Initial psychophysical evidence. Vision Research, 1968, 8, 445-450,

Sekuler, R. W., \& Ganz, L. Aftereffect of seen motion with a stabilized retinal image. Science, 1963. 139, 419-420.

Wundt, W. Grundsiige der Physiologischen psy. chologie. Leipzig, Germany: Engelmann, 1874.

(Received March 10, 1975) 